\title{
Estimation of musculotendon parameters for scaled and subject specific musculoskeletal models using an optimization technique
}

\author{
Authors: $\quad$ Luca Modenese ${ }^{1,2,3}$, Elena Ceseracciu ${ }^{4}$, Monica Reggiani $^{4}$, David G, Lloyd ${ }^{1}$ \\ Affiliations: $\quad{ }^{1}$ Centre for Musculoskeletal Research, School of Allied Health Sciences, \\ Menzies Health Institute Queensland, Griffith University, Southport, Australia \\ ${ }^{2}$ Department of Mechanical Engineering, University of Sheffield \\ ${ }^{3}$ INSIGNEO institute for in silico medicine, University of Sheffield, United \\ Kingdom \\ ${ }^{4}$ Department of Management and Engineering, University of Padua, Vicenza, \\ Italy \\ Article type: $\quad$ Original Article \\ Word count: 247 (abstract) / 3695(manuscript)
}

Keywords: optimal fiber length, tendon slack length, scaling, subject specific, muscle models, hip joint, muscle-tendon parameters, parameter optimization

Corresponding author:

Luca Modenese

Email: $\quad$ 1.modenese@sheffield.ac.uk

Address: $\quad$ INSIGNEO institute for in silico medicine, Department of Mechanical Engineering, Pam Liversidge Building, Mappin Street University of Sheffield

S1 3JD Sheffield, United Kingdom 


\begin{abstract}
A challenging aspect of subject specific musculoskeletal modeling is the estimation of muscle parameters, especially optimal fiber length and tendon slack length. In this study, the method for scaling musculotendon parameters published by Winby et al. (2008), Journal of Biomechanics 41, 1682-1688, has been reformulated, generalized and applied to two cases of practical interest: 1) the adjustment of muscle parameters in the entire lower limb following linear scaling of a generic model and 2) their estimation "from scratch" in a subject specific model of the hip joint created from medical images. In the first case, the procedure maintained the muscles' operating range between models with mean errors below $2.3 \%$ of the reference model normalized fiber length value. In the second case, a subject specific model of the hip joint was created using segmented bone geometries and muscle volumes publicly available for a cadaveric specimen from the Living Human Digital Library (LHDL). Estimated optimal fiber lengths were found to be consistent with those of a previously published dataset for all 27 considered muscle bundles except gracilis. However, computed tendon slack lengths differed from tendon lengths measured in the LHDL cadaver, suggesting that tendon slack length should be determined via optimization in subject-specific applications. Overall, the presented methodology could adjust the parameters of a scaled model and enabled the estimation of muscle parameters in newly created subject specific models. All data used in the analyses are of public domain and a tool implementing the algorithm is available at https://simtk.org/home/opt_muscle_par.
\end{abstract}

NOTE FOR REVIEWERS: A zip file including the models and Matlab scripts used in this investigation has been submitted as supplementary material together with the manuscript. The scripts are designed to permit easy reproduction of the results and they will be part of the package downloadable after publication. 


\section{Introduction}

Musculoskeletal modeling is a computational technique that allows non-invasive estimation of internal joint loads and muscle forces, yielding more accurate results when personalized musculoskeletal geometry is used in the simulations (Gerus et al., 2013; Marra et al., 2015). Although largely unexplored, musculoskeletal models also have the potential of investigating predictive if-then scenarios such as post-operative functional outcomes after virtual interventions (Delp et al., 2007; Fregly, 2009).

However, applications of this kind rely on muscle-actuated simulations based on forward dynamics that are currently challenging to generate when using subject specific models derived from medical images. One of the reasons is that while personalized bone geometries and joint parameters can be obtained from segmented medical images, musculotendon parameters currently cannot be easily measured or estimated, although improvements in imaging and segmentation technology might change the situation in the future (Blemker et al., 2007). For example, if a Hill-type muscle model as proposed by Zajac (1989) is adopted, the maximum isometric force $F_{i s o}^{m}$ can be calculated from muscle volumes (Hainisch et al., 2012; Handsfield et al., 2014), but optimal fiber length $l_{o}^{m}$ and tendon slack length $l_{s}^{t}$ are parameters that are difficult to estimate and highly influence the model force outputs (Scovil and Ronsky, 2006; Redl et al., 2007).

Few algorithms are available in the literature to estimate these parameters and they can be divided into anthropometric and functional approaches, depending if they rely just on skeletal dimensions or also on additional measurements. Previously proposed anthropometric algorithms assume $l_{o}^{m}$ to be known (Manal and Buchanan, 2004) or match the muscle fiber operating ranges of a generic model to a scaled model (Winby et al., 2008). Musculoskeletal software such as OpenSim (Delp et al., 2007) and AnyBody (Damsgaard et al., 2006) also use anthropometric methods. In OpenSim the ratio of $l_{o}^{m}$ to $l_{s}^{t}$ of the generic model is preserved in the scaled model, while in AnyBody $l_{s}^{t}$ is adjusted in order to maintain the joint angle at which muscle force peaks. On the other hand, functional methods (Hatze, 1981; Garner and Pandy, 2003; Lloyd and Besier, 2003) generally require collection of a relatively large amount of experimental data, which may not always be feasible. Furthermore, other approaches have only been tested with simulated data (Van Campen et al., 2014) or assume muscle forces known from a previous optimization procedure (Ojeda and Mayo, 2013), so evidence of their performance in experimental conditions is still lacking. 
A common shortcoming of all anthropometric methods is that they focus on a single joint, so considering muscle spanning multiple degrees of freedom (DOFs) as mono-articular (Van Campen et al., 2014), not explicitly treating the case of multi-articular muscles (Manal and Buchanan, 2004) or approaching the issue in a simplified way, for instance just considering motion in the sagittal plane (Winby et al., 2008). A further limitation is that they have only been used in linearly scaled adaptations of existing generic models, and never to generate musculotendon parameters in subject specific models built from medical images.

The two fold aim of this paper was to develop an anthropometric algorithm so that musculotendon parameters can be 1) optimized for a complete three dimensional lower limb model (and not just for the muscles crossing a single joint) and 2) estimated ex novo for a subject specific musculoskeletal model generated based on medical images. The first aim was undertaken by generalizing the algorithm of Winby et al. (2008), while the second objective used a new model of the hip, generated from medical images available through the Living Human Digital Library (LHDL) dataset (Viceconti et al., 2008). All presented examples are developed from freely available resources and therefore are easily reproducible (see Appendix for details).

\section{Methods}

\subsection{Dimensionless muscle model}

A dimensionless Hill-type muscle model as proposed by Zajac (1989) (Figure 1) uses normalized functions to define the active and passive muscle force-length curves, forcevelocity curve and tendon force-strain curve and needs five parameters to be defined: optimal fiber length $l_{o}^{m}$, tendon slack length $l_{s}^{t}$, maximum isometric force $F_{i s o}^{m}$, pennation angle at optimal fiber length $\alpha_{o}$ and maximum contraction velocity $v_{\max }$. Equilibrium of the musculotendon unit is obtained when the tendon and the fiber forces along the tendon direction are equal.

\subsection{Describing the dimensionless operating range}

The length of a musculotendon actuator $l^{m t}$ can be calculated from the muscle length $l^{m}$ and tendon length $l^{t}$ as follows (Figure 1A):

$$
l^{m t}=l^{m} \cos \alpha+l^{t}
$$


where $\alpha$ is the pennation angle at that specific muscle length, calculated assuming constant muscle thickness, as:

$$
\alpha=\operatorname{asin}\left(\frac{l_{o}^{m} \sin \boldsymbol{\alpha}_{\boldsymbol{o}}}{\boldsymbol{l}^{\boldsymbol{m}}}\right)
$$

Following Zajac (1989) we defined the normalized fiber length as:

$$
\tilde{l}^{m}=\frac{l^{m}}{l_{o}^{m}}
$$

and we decided to define a normalized tendon length ( $\varepsilon^{t}$ being tendon strain) as:

$$
\tilde{l}^{t}=\frac{l^{t}}{l_{s}^{t}}=\left(1+\varepsilon^{t}\right)
$$

Using normalized coefficients, the musculotendon length can now be expressed as $\boldsymbol{f}\left(\boldsymbol{l}_{\boldsymbol{o}}^{\boldsymbol{m}}, \boldsymbol{l}_{\boldsymbol{s}}^{\boldsymbol{t}}\right)$ :

$$
\boldsymbol{l}^{m \boldsymbol{t}}=\left(\tilde{\boldsymbol{l}}^{m} \cos \alpha\right) \boldsymbol{l}_{\boldsymbol{o}}^{\boldsymbol{m}}+\tilde{\boldsymbol{l}}^{t} \boldsymbol{l}_{\boldsymbol{s}}^{t}
$$

If the normalized coefficients are calculated from $\boldsymbol{l}^{\boldsymbol{m}}$ and $\boldsymbol{l}^{\boldsymbol{t}}$ in a musculotendon unit equilibrated for isometric contraction at maximum activation, Eq. 5 ensures static equilibrium between the tendon and (active plus passive) muscle force of any muscle actuator of length $\boldsymbol{l}^{\boldsymbol{m} \boldsymbol{t}}$ whose $\boldsymbol{l}_{\boldsymbol{o}}^{\boldsymbol{m}}$ and $\boldsymbol{l}_{\boldsymbol{s}}^{\boldsymbol{t}}$ satisfy that equation. For example, an equilibrated condition for a muscle with null pennation angle is represented by the red dashed lines in Figure 1B. The entire operating range and isometric force generating capability of a muscle can be described by its normalized coefficients calculated as a function of the $\overrightarrow{\boldsymbol{q}}$ joint angles spanned by the muscle in the model.

\subsection{Musculotendon parameters estimation}

Similar to Winby et al. (2008), the proposed method aimed to map the normalized muscle operating conditions of an existing "reference model", whose muscle parameters were assumed to be physiologically valid, onto a "target model" of different anthropometric dimensions for equivalent joint configurations. For the examples presented in this paper (see sections 2.4 and 2.5) the reference and target models are described in Table 1. The algorithm, represented as flow chart in Figure 2A, consists of the following steps, applied to each muscle included in the model:

1) In the reference model, the $\boldsymbol{N}_{\boldsymbol{q}}$ coordinates (or degrees of freedom) $\overrightarrow{\boldsymbol{q}}$ spanned by the musculotendon actuator were uniformly sampled using $\boldsymbol{n}_{\boldsymbol{d o f}}$ points per coordinate. As all combinations of generalized coordinates were considered, a set of $\boldsymbol{n}=\left(\boldsymbol{n}_{\boldsymbol{d o f}}\right)^{\boldsymbol{N}_{\boldsymbol{q}}}$ 
total model poses was generated, in which the considered muscle was equilibrated for a maximum isometric contraction (i.e. activation $=1$ ).

2) For each pose $\boldsymbol{i}=\mathbf{1}, \mathbf{2}, \ldots, \boldsymbol{n}$ of the reference model, the vectors of pennation angles $\boldsymbol{\alpha}_{i, \boldsymbol{r} \boldsymbol{f}}$, normalized fiber lengths $\tilde{\boldsymbol{l}}_{\boldsymbol{i}, \boldsymbol{r} \boldsymbol{e}}$ and normalized tendon lengths $\tilde{\boldsymbol{l}}_{\boldsymbol{i}, \boldsymbol{r} \boldsymbol{e} \boldsymbol{f}}^{t}$ were calculated using Eqs 2,3,4. Normalized fiber lengths outside the range of 0.5 and 1.5 or causing pennation angles approaching 90 degrees were excluded.

3) The musculotendon lengths $\boldsymbol{l}_{\text {targ }}^{\boldsymbol{m} t}$ were calculated in the target model for the same joint configurations used in the reference model at step 1.

4) Imposing Eq. 5 for all poses, the following $\boldsymbol{n} \boldsymbol{x} \mathbf{2}$ linear system was obtained and could be solved in a least square sense, so obtaining the unknown values of $\boldsymbol{l}_{\boldsymbol{o} \text {,targ }}^{\boldsymbol{m}}$ and $\boldsymbol{l}_{\boldsymbol{s}, \boldsymbol{t a r g}}$.

$$
\left[\begin{array}{c}
\mathbf{l}_{1}^{\mathrm{mt}} \\
\mathbf{l}_{2}^{\mathbf{m t}} \\
\vdots \\
\mathrm{l}_{\mathrm{n}}^{\mathrm{mt}}
\end{array}\right]_{\operatorname{targ}}=\left[\begin{array}{cc}
\tilde{\mathrm{I}}_{1}^{\mathrm{m}} \cos \alpha_{1} & \tilde{\mathrm{I}}_{1}^{\mathrm{t}} \\
\tilde{\mathrm{I}}_{2}^{\mathrm{m}} \cos \alpha_{2} & \tilde{\mathrm{I}}_{2}^{\mathrm{t}} \\
\vdots & \vdots \\
\tilde{\mathrm{I}}_{\mathrm{n}}^{\mathrm{m}} \cos \alpha_{\mathrm{n}} & \tilde{\mathrm{I}}_{\mathrm{n}}^{\mathrm{t}}
\end{array}\right]_{\mathrm{ref}}\left[\begin{array}{c}
\mathrm{l}_{\mathrm{o}}^{\mathrm{m}} \\
\mathrm{l}_{\mathrm{s}}^{\mathrm{t}}
\end{array}\right]_{\operatorname{targ}}
$$

5) If the system yielded non physiological results, such as $\mathrm{l}_{\mathrm{s}, \mathrm{targ}}^{\mathrm{t}}<0$, the tendon fraction of $\mathrm{l}_{\mathrm{targ}}^{\mathrm{mt}}$ was temporarily fixed at the same proportion of the reference model, $\mathrm{l}_{\mathrm{o}, \operatorname{targ}}^{\mathrm{m}}$ estimated by solving Eq. 6 and finally $\mathrm{l}_{\mathrm{s} \text {,targ }}^{\mathrm{t}}$ re-computed from the same equation using the new value of $\mathrm{l}_{\mathrm{o}, \text { targ }}^{\mathrm{m}}$.

The algorithm was implemented using the application programming interface of OpenSim 3.2 (Delp et al., 2007).

\subsection{Example 1: A linearly scaled model}

This first example of muscle parameter optimization used a generic lower limb musculoskeletal model based on Delp et al. (1990) and available in the OpenSim distribution as 'gait2392'. The model was scaled using the proportions published by Hamner et al. (2010); the joint ranges of motion were limited according to the running kinematics reported in the same study. The linear system described in Eq. 6 was solved for the 46 muscles of the left leg and the mapping results evaluated as described in Section 2.6. The dimensionless muscle model included in gait2392 is based on the implementation of Thelen (2003).

\subsection{Example 2: A musculoskeletal model from MRI images}

A musculoskeletal model of the right hip was generated using the publicly available LHDL dataset (Viceconti et al., 2008), which includes bone geometries and muscle volume 
meshes segmented respectively from images of computed tomography (CT) and magnetic resonance imaging (MRI) for a cadaveric specimen (female, 81 years old, 1,67 m, $63 \mathrm{~kg}$ ). The hip joint centre was located by fitting a sphere in a least squares sense to the femoral head mesh. Muscle attachment points and muscle paths were defined in NMSBuilder (Valente et al., 2014) based on the available three dimensional volume reconstructions (see selected muscles in Figure 3). A number of musculotendon paths for each muscle were also spatially reconstructed during dissection (differentiating between fiber and tendon) using pins located in the surface of the muscles and digitized through snapshot-stereophotogrammetry, as described in the LHP data collection white paper $^{1}$; this geometrical information was used as an additional help when defining the muscle geometries. An OpenSim model was produced that included 19 muscles (represented by 27 individual bundles) spanning a three DOFs (ball and socket) hip joint. The musculoskeletal model was limited to the hip joint because segmented muscle volumes, and therefore well-defined musculotendon paths, were only available for that anatomical region; the knee joint was consequently locked in the reference position. The lower limb model published by Arnold et al. (2010) based on the cadaveric measurement of Ward et al. (2009), was used as reference model and mapped onto the MRI-based model, limiting the range of motion to joint angles (Table 1) consistent with normal walking (Kadaba et al., 1990). Both models used a dimensionless muscle model based on Schutte et al. (1993) and the pennation angles of the reference model were adopted in the target.

\subsection{Assessment of muscle mapping and estimated musculotendon parameters}

To determine the optimal number of model poses, $n=\left(n_{d o f}\right)^{N_{q}}$, required by the algorithm, and to assess the robustness of the methodology, a sensitivity analysis of the calculated muscle parameters to the number of evaluation points, $\mathrm{n}_{\mathrm{dof}}$, was investigated for both examples. Convergence was evaluated through the percentage of variation of the muscle parameters when calculated using $n_{\text {dof }}$ discretization points with respect to $n_{d o f}-1$, with $\mathrm{n}_{\mathrm{dof}}$ varying between five and fifteen. To assess how well the reference model's muscle fiber lengths mapped onto the target model we compared the $\tilde{l}^{m}(\vec{q})$ values, obtained from the optimized models, with the $\tilde{l}_{\text {ref }}^{m}(\vec{q})$ values, which were considered "correct". This comparison was performed using the values calculated at ten fixed evaluation points per degree of

\footnotetext{
${ }^{1}$ Available at https://www.biomedtown.org/biomed town/LHDL/Reception/lhp-publicrepository/public_D/plfng_view. See also Moiseev et al. (2008) and Figure 7 of Van Sint Jan (2005).
} 
freedom and employed three metrics: 1) root mean squared error (RMSE) as a measure of global fitting, 2) correlation coefficients as indicators of the shape similarity between muscle fiber length operating profiles and 3) mean error, expressed as percentage of $\tilde{l}_{\text {ref }}^{m}(\vec{q})$.

For the model based on medical images, optimal fiber lengths obtained by optimization were compared against the cadaveric measurements of Ward et al. (2009), as the LHDL specimen was compatible with the population demographics of that study (21 specimens of which 12 females, age: $83 \pm 9$ years, height: $168.4 \pm 9.3 \mathrm{~cm}$, mass: $82.7 \pm 15.3 \mathrm{~kg}$ ). Estimated tendon slack lengths were compared to those calculated by Arnold et al. (2010) and the range of tendon lengths measured during the dissection of the LHDL specimen. The assessment procedure is represented as a flow chart in Figure 2B.

\section{Results}

For both examples variations of $\mathrm{l}_{\mathrm{o}, \text { targ }}^{\mathrm{m}}, \mathrm{l}_{\mathrm{s}, \text { targ }}^{\mathrm{t}}$ and RMSE for each muscle (Figure 4) decreased asymptotically with increasing number of the sampling points, $\mathrm{n}_{\text {dof }}$. For $\mathrm{n}_{\mathrm{dof}}>$ 10 all variations were smaller than $4 \%$, suggesting that convergence was achieved. Results are presented for $n_{d o f}=10$, which were identical to $n_{d o f}=15$.

Muscle parameter optimization for the linearly scaled model yielded good mapping, with the largest mean error of $2.3 \%$ and RMSE of 0.019 found for biceps femoris caput longum. As expected, muscles spanning multiple joints were associated with larger mapping errors (Figure 5). The non-linear scaling of the musculotendon lengths caused changes in all muscle parameters; the largest variation was $18 \%$ for optimal fiber length (peroneus brevis) and $25 \%$ for tendon slack length (adductor brevis). Correlation coefficients between reference and target $\tilde{\mathrm{l}}_{\text {ref }}(\overrightarrow{\mathrm{q}})$ curves were always larger than $0.99(\mathrm{p}<1 \mathrm{e}-5)$.

When muscle operating ranges $\mathrm{l}_{\text {ref }}^{\mathrm{m}}(\overrightarrow{\mathrm{q}})$ were mapped to the MRI-based model, estimated optimal fiber lengths were within two standard deviations of the measurements reported by Ward et al. (2009) for all muscles, except gracilis (Figure 6, first row). The adjusted tendon slack lengths were similar to Arnold's et al. (2010) estimates, while mostly outside the range of tendon length measurements taken on the LHDL specimen (Figure 6, second row). The mean fitting errors were larger than in the first example, reaching $23 \%$ for quadratus femoris. The fiber operating ranges of reference and target models were strongly correlated (range: 0.68-0.99, p<1e-5). 


\section{Discussion}

An optimization algorithm aiming to estimate subject-specific musculotendon parameters was presented and applied to two cases of interest in musculoskeletal modeling: the adjustment of musculotendon parameters after linear scaling of a generic lower limb model and their estimation "from scratch" in the case of a model generated entirely from medical images.

In the first example, the proposed methodology was able to optimize the muscle parameters yielding very good mapping metrics using 10 evaluation points per degree of freedom. The variations of muscle parameters (unconstrained in the algorithm) were comparable with the variability reported by Ward et al. (2009) for optimal fiber lengths (standard deviation ranging from $11 \%$ of the mean $\mathrm{l}_{\mathrm{o}}^{\mathrm{m}}$ value for sartorius, up to $27 \%$ of mean value for biceps femoris caput longum and semimembranosus).

Muscle parameters adjustment in a linearly scaled model can serve multiple purposes, for instance it has been shown to significantly improve accuracy of static EMG driven models (Menegaldo and Oliveira, 2009), and it is necessary due to the nonlinear relationship between musculotendon lengths and segment dimensions. The entity of these adjustments is influenced both by the accuracy and degree of non uniformity of the scaling operation, which can be quantified by a proportionality index (Winby et al., 2008). In the presented example this index resulted consistent $(0.062$, see Table 1) with those of the ten subjects $(0.087 \pm 0.069)$ analyzed in Winby et al. (2008), suggesting similar variations of scaled model's proportions from the generic model.

The second example estimated musculotendon parameters for a newly generated subject specific model. Although the MRI images were collected on a cadaveric specimen, this model was generated from geometry data that could be obtained in vivo through segmentation of medical images. As shown in Figure 6, estimated optimal fiber lengths are compatible with the measurements of Ward et al. (2009) for all muscles except gracilis, for which the calculation of $\mathrm{l}_{\mathrm{o}}^{\mathrm{m}}$ may have been affected by ignoring knee mobility in the optimization process. In general, consistency between the reference and target model in the definition of muscle geometry is desirable, especially for muscles with large attachment areas for which alternative definitions of musculotendon paths are possible, in order to avoid mapping configurations leading to overstretched or slack musculotendon units.

The majority of muscles' tendon slack lengths did not fall within the range of tendon lengths measured in the LHDL specimen (Figure 6, second row). This inconsistency can 
partially be explained by the measurements being taken on the specimen in only the anatomical position, where the tendons were not necessarily slack. Furthermore, the musculoskeletal model includes a limited number of bundles to represent complex threedimensional muscle geometries, which results in different total lengths $\mathrm{l}^{\mathrm{mt}}$ between the measured and modeled musculotendon fibers; an average difference of $3.3( \pm 3.1) \mathrm{cm}$ was found in the specimen pose, due in part to the measurements being taken on the surface of the muscle bellies (Moiseev et al., 2008) and in part to errors introduced by straight line muscle representation (Jensen and Davy, 1975) despite careful definition of lines of action. This interpretation of the results, consistent with the findings of Klein Breteler et al. (1999) at the upper extremity, suggests that $\mathrm{l}_{\mathrm{s}}^{\mathrm{t}}$ might need to be adjusted via optimization even if tendon and fiber lengths could be measured in vivo due to the model geometrical errors. The effect of muscle volumetric representation, e.g. through personalized wrapping surfaces (van Arkel et al., 2013), on muscle parameters estimation should be investigated in the future.

In our simulations, non-physiological muscle parameter estimation $\left(\mathrm{l}_{\mathrm{s}, \operatorname{targ}}^{\mathrm{t}}<0\right)$ occurred only for pectineus $\left(\mathrm{l}_{\mathrm{s}}^{\mathrm{t}}=1 \mathrm{~mm}\right.$ in the reference model). This was due to an almost constant tendon length throughout the range of motion malconditioning the matrix of the normalized coefficient in Eq. 6. Based on our tests, performing the mapping procedure on a range of motion appropriate for the task(s) of interest is recommended. The presented method could also be applied using sub-maximal isometric contractions in step 1). When tested with activations equal to 0.5 and 0 , for the scaled model the algorithm yielded musculotendon parameters less than $0.5 \%$ different with respect to the maximal activation case. Similar results occurred in the MRI-based model, although the $\mathrm{l}_{\mathrm{s}}^{\mathrm{t}}$ of three muscles (gluteus maximus, gracilis and sartorius) changed by more than $5 \%$. These muscles, like the aforementioned pectineus, are stiff musculotendon units $\left(\mathrm{l}_{\mathrm{s}}^{\mathrm{t}}<\mathrm{l}_{\mathrm{o}}^{\mathrm{m}}\right)$ (Zajac, 1989), meaning that their tendon does not stretch significantly, so influencing the optimization results for $\mathrm{l}_{\mathrm{s}}^{\mathrm{t}}$.

One of the aims of the presented methodology was to offer an appropriate estimation of $\mathrm{l}_{\mathrm{o}}^{\mathrm{m}}$ and $\mathrm{l}_{\mathrm{s}}^{\mathrm{t}}$ in subject specific models built from medical images to enable muscle-actuated simulations, as the other muscle parameters can already be identified. Pennation angle can be taken from the literature as it has small influence on muscle force (Zajac, 1989), $\mathrm{v}_{\max }$ is generally assumed equal to $10 \mathrm{l}_{\mathrm{o}}^{\mathrm{m}} / \mathrm{s}$ (Zajac, 1989) and $\mathrm{F}_{\mathrm{iso}}^{\mathrm{m}}$ is proportional to the physiological cross sectional area (PCSA), calculated as PCSA $=\left(\operatorname{Vol} * \cos \alpha_{o}\right) / l_{0}^{m}($ Ward et al., 2009), where Vol is the muscle volume obtained through segmentation for clinical populations (Hainisch et al., 2012) or using regression equations for young healthy subjects (Handsfield 
et al., 2014). Further work is necessary to assess the proposed algorithm when applied on muscles crossing other joints, to investigate its potential use in combination with experimental measurements of muscle architecture parameters, e.g. Rubenson et al. (2012), or for adjusting cadaveric measurements (Klein Horsman et al., 2007) before inclusion in musculoskeletal models (Modenese et al., 2011; Modenese and Phillips, 2012; Modenese et al., 2015). We expect differences in muscle function with respect to the reference model to be small when the normalized fiber lengths in the reference and target models are similar for the same joint angles in isometric contraction conditions. However, in dynamic simulations with variable muscle activations the effect of using simply scaled versus optimized parameters on musculotendon function needs further assessment.

The proposed algorithm has several advantages over the method recommended by Winby et al. (2008) from which it was developed. First, our method has been formulated to be generic, in that it considers the operating range of all muscles of the lower limb model for all anatomical motion planes and not just the muscles spanning the knee joint in the sagittal plane. Subsequently, our implementation could be applied to other anatomical regions, e.g. the shoulder, if a reference model is available. Second, our algorithm fully exploits the dimensionless Hill-type muscle model proposed by Zajac (1989) by using normalized coefficients that ensure muscle-tendon isometric equilibrium while avoiding the need to solve muscle contraction dynamics within the optimization procedure, which makes the procedure robust and fast to compute. Using normalized coefficients also means the method can be applied with all dimensionless musculotendon modeling implementations (Schutte et al., 1993; Lloyd and Besier, 2003; Thelen, 2003; Millard et al., 2013). Maintaining muscle model consistency in reference and target model is however recommended.

One limitation of the presented method concerns the underlying assumption that any muscle's range of operation, i.e. force-joint angle relationship, is the same across individuals. Although there is evidence that muscles mainly work on the ascending limb of the forcelength relationship (Burkholder and Lieber, 2001) and that sarcomeres (in the muscle fibers of immobilize mice) can rearrange their number to maintain optimal filament overlap (Williams and Goldspink, 1978), there is not enough experimental evidence to assume a functional scaling criterion for $\mathrm{l}_{\mathrm{o}}^{\mathrm{m}}$ between individuals. Conversely, it has been shown that muscle architecture in adults does not scale linearly with skeletal dimensions (Ward et al., 2005), so our approach is a justified enhancement compared to the linear anthropometric scaling methods currently implemented in existing musculoskeletal software. The proposed method requires a reference musculoskeletal model implementing physiological muscle 
operating ranges to be mapped into the target model. This limitation was overcome in the first example by using the OpenSim model "gait2392" based on Delp et al. (1990), while in the second example a lower limb model (Arnold et al., 2010) including the relationships between joint angles and sarcomere lengths as measured in 21 cadavers by Ward et al. (2009) was adopted. A further limitation is that muscle operating ranges of the reference models are considered representative of average healthy individuals (although obtained from elderly specimens) and should not be used for different athletics groups (Abe et al., 2000; Kumagai et al., 2000; Lee and Piazza, 2009), or more importantly different clinical populations, for example altered musculotendon architecture (Barber et al., 2012) or fascicle lengths and passive torque-joint angle relationships (Barber et al., 2011). In these cases, further functionally based optimization procedures using experimental measurements can be used for adjusting the anthropometrically scaled models produced with the presented technique.

\section{Conclusion}

The recommended method of Winby et al. (2008) was reformulated and generalized so that it can be used to adjust muscle parameters of any musculoskeletal model when linearly scaled from a generic reference model including valid muscle parameters. This generalized method can also be used to estimate optimal fiber lengths and tendon slack lengths in new subject specific models created from medical images. In this second application, estimated parameters for hip spanning muscles were shown to be consistent with previously published cadaveric measurements, so ensuring the validity of the proposed methodology in the perspective of enabling muscle-actuated simulations using fully personalized models. A Matlab tool and OpenSim plugin implementing the presented algorithm will be made available at https://simtk.org/home/opt_muscle_par.

\section{Conflict of interest}

None of the authors have any financial or personal conflict of interest with regard to this study.

\section{Acknowledgements}

Luca Modenese wants to thank Ananthamaran Gopalakrishnan and Claudio Pizzolato for the discussions about the topic of this manuscript and more generally about muscle models. The authors are also grateful to David Saxby and Alice Mantoan for the helpful comments on the 
manuscript and for testing preliminary versions of the Matlab toolbox. Funding for this study was provided by Griffith University Areas of Strategic Investment (to DGL), Brisbane Royal Children's Hospital Private Practice Research Fund (to DGL), and European Union Framework Programme 7, EU-FP7 grant BioMot (p. no. 611695), (to MR).

\section{Appendix A}

The original models and experimental data used in the present publication are available at:

Example1: https://simtk.org/home/runningsim

Example2 (reference model): https://simtk.org/home/lowlimbmodel09

Example2 (data used to build the subject specific model): https://www.physiomespace.com

\section{References}

Abe, T., Kumagai, K. and Brechue, W. F., 2000. Fascicle length of leg muscles is greater in sprinters than distance runners. Medicine and science in sports and exercise 32, 11251129.

Arnold, E., Ward, S., Lieber, R. and Delp, S., 2010. A Model of the Lower Limb for Analysis of Human Movement. Annals of biomedical engineering 38, 269-279.

Barber, L., Barrett, R. and Lichtwark, G., 2011. Passive muscle mechanical properties of the medial gastrocnemius in young adults with spastic cerebral palsy. Journal of Biomechanics 44, 2496-2500.

Barber, L., Barrett, R. and Lichtwark, G., 2012. Medial gastrocnemius muscle fascicle active torque-length and Achilles tendon properties in young adults with spastic cerebral palsy. Journal of Biomechanics 45, 2526-2530.

Blemker, S. S., Asakawa, D. S., Gold, G. E. and Delp, S. L., 2007. Image-based musculoskeletal modeling: applications, advances, and future opportunities. Journal of Magnetic Resonance Imaging 25, 441-451.

Burkholder, T. J. and Lieber, R. L., 2001. Sarcomere length operating range of vertebrate muscles during movement. Journal of Experimental Biology 204, 1529-1536.

Damsgaard, M., Rasmussen, J., Christensen, S. T., Surma, E. and de Zee, M., 2006. Analysis of musculoskeletal systems in the AnyBody Modeling System. Simulation Modelling Practice and Theory 14, 1100-1111.

Delp, S. L., Loan, J. P., Hoy, M. G., Zajac, F. E., Topp, E. L. and Rosen, J. M., 1990. An interactive graphics-based model of the lower extremity to study orthopaedic surgical procedures. IEEE Transactions on Biomedical Engineering 37, 757-767. 
Delp, S. L., Anderson, F. C., Arnold, A. S., Loan, P., Habib, A., John, C. T., Guendelman, E. and Thelen, D. G., 2007. OpenSim: open-source software to create and analyze dynamic simulations of movement. IEEE Transactions on Biomedical Engineering 54, 1940-1950.

Fregly, B. J., 2009. Design of optimal treatments for neuromusculoskeletal disorders using patient-specific multibody dynamic models. International journal for computational vision and biomechanics 2, 145.

Garner, B. A. and Pandy, M. G., 2003. Estimation of Musculotendon Properties in the Human Upper Limb. Annals of biomedical engineering 31, 207-220.

Gerus, P., Sartori, M., Besier, T. F., Fregly, B. J., Delp, S. L., Banks, S. A., Pandy, M. G., D'Lima, D. D. and Lloyd, D. G., 2013. Subject-specific knee joint geometry improves predictions of medial tibiofemoral contact forces. Journal of Biomechanics 46, 27782786.

Hainisch, R., Gfoehler, M., Zubayer-Ul-Karim, M. and Pandy, M. G., 2012. Method for determining musculotendon parameters in subject-specific musculoskeletal models of children developed from MRI data. Multibody System Dynamics 28, 143-156.

Hamner, S. R., Seth, A. and Delp, S. L., 2010. Muscle contributions to propulsion and support during running. Journal of Biomechanics 43, 2709-2716.

Handsfield, G. G., Meyer, C. H., Hart, J., Abel, M. and Blemker, S. S., 2014. Relationships of 35 Lower Limb Muscles to Height and Body Mass Quantified Using MRI. Journal of Biomechanics 47, 631-638.

Hatze, H., 1981. Estimation of myodynamic parameter values from observations on isometrically contracting muscle groups. European Journal of Applied Physiology and Occupational Physiology 46, 325-338.

Jensen, R. H. and Davy, D. T., 1975. An investigation of muscle lines of action about the hip: A centroid line approach vs the straight line approach. Journal of Biomechanics 8, 103-110.

Kadaba, M. P., Ramakrishnan, H. K. and Wootten, M. E., 1990. Measurement of lower extremity kinematics during level walking. Journal of Orthopaedic Research 8, 383-392.

Klein Breteler, M. D., Spoor, C. W. and Van der Helm, F. C. T., 1999. Measuring muscle and joint geometry parameters of a shoulder for modeling purposes. Journal of Biomechanics 32, 1191.

Klein Horsman, M. D., Koopman, H. F., van der Helm, F. C., Prose, L. P. and Veeger, H. E., 2007. Morphological muscle and joint parameters for musculoskeletal modelling of the lower extremity. Clinical Biomechanics 22, 239-247.

Kumagai, K., Abe, T., Brechue, W. F., Ryushi, T., Takano, S. and Mizuno, M., 2000. Sprint performance is related to muscle fascicle length in male $100-\mathrm{m}$ sprinters. Journal of Applied Physiology 88, 811-816. 
Lee, S. S. and Piazza, S. J., 2009. Built for speed: musculoskeletal structure and sprinting ability. Journal of Experimental Biology 212, 3700-3707.

Lloyd, D. G. and Besier, T. F., 2003. An EMG-driven musculoskeletal model to estimate muscle forces and knee joint moments in vivo. Journal of Biomechanics 36, 765-776.

Manal, K. and Buchanan, T. S., 2004. Subject-Specific Estimates of Tendon Slack Length: A Numerical Method. Journal of applied Biomechanics 20, 195-203.

Marra, M. A., Vanheule, V., Fluit, R., Koopman, B. H., Rasmussen, J. and Verdonschot, N., 2015. A Subject-Specific Musculoskeletal Modeling Framework to Predict in Vivo Mechanics of Total Knee Arthroplasty. Journal of Biomechanical Engineering 137, 020904.

Menegaldo, L. L. and Oliveira, L. F. d., 2009. Effect of muscle model parameter scaling for isometric plantar flexion torque prediction. Journal of Biomechanics 42, 2597-2601.

Millard, M., Uchida, T., Seth, A. and Delp, S. L., 2013. Flexing Computational Muscle: Modeling and Simulation of Musculotendon Dynamics. Journal of Biomechanical Engineering 135, 021005-021005.

Modenese, L., Phillips, A. T. M. and Bull, A. M. J., 2011. An open source lower limb model: Hip joint validation. Journal of Biomechanics 44, 2185-2193.

Modenese, L. and Phillips, A., 2012. Prediction of hip contact forces and muscle activations during walking at different speeds. Multibody System Dynamics 28, 157168.

Modenese, L., Phillips, A. T. and Bull, A. M. J., 2015. Letter to the Editor: In response to "Consistency Among Musculoskeletal Models: Caveat Utilitor". Annals of biomedical engineering 43, 1052-1054.

Moiseev, F., Sholukha, V., Snoeck, O., Salvia, P., Rooze, M. and Van Sint Jan, S., 2008. Registration of soft tissue morphology with skeletal morphology and kinematics. Journal of Biomechanics 41, Supplement 1, S204.

Ojeda, J. and Mayo, J., 2013. A New Approach to Estimate a Subject-Specific Set of Muscle Parameters. In: Viadero, F. and Ceccarelli, M. (Eds), New Trends in Mechanism and Machine Science. Springer Netherlands, pp. 479-485.

Redl, C., Gfoehler, M. and Pandy, M. G., 2007. Sensitivity of muscle force estimates to variations in muscle-tendon properties. Human Movement Science 26, 306-319.

Rubenson, J., Pires, N. J., Loi, H. O., Pinniger, G. J. and Shannon, D. G., 2012. On the ascent: the soleus operating length is conserved to the ascending limb of the force-length curve across gait mechanics in humans. The Journal of Experimental Biology 215, 3539-3551. 
Schutte, L. M., Rodgers, M. M., Zajac, F. E. and Glaser, R. M., 1993. Improving the efficacy of electrical stimulation-induced leg cycle ergometry: an analysis based on a dynamic musculoskeletal model. IEEE Transactions on Rehabilitation Engineering 1, 109-125.

Scovil, C. Y. and Ronsky, J. L., 2006. Sensitivity of a Hill-based muscle model to perturbations in model parameters. Journal of Biomechanics 39, 2055-2063.

Thelen, D. G., 2003. Adjustment of Muscle Mechanics Model Parameters to Simulate Dynamic Contractions in Older Adults. Journal of Biomechanical Engineering 125, 70 77.

Valente, G., Pitto, L., Testi, D., Seth, A., Delp, S. L., Stagni, R., Viceconti, M. and Taddei, F., 2014. Are Subject-Specific Musculoskeletal Models Robust to the Uncertainties in Parameter Identification? PLoS ONE 9, e112625.

van Arkel, R. J., Modenese, L., Phillips, A. and Jeffers, J. R., 2013. Hip Abduction Can Prevent Posterior Edge Loading of Hip Replacements. Journal of Orthopaedic Research 31, 1172-1179.

Van Campen, A., Pipeleers, G., De Groote, F., Jonkers, I. and De Schutter, J., 2014. A new method for estimating subject-specific muscle-tendon parameters of the knee joint actuators: a simulation study. International Journal for Numerical Methods in Biomedical Engineering 30, 969-987.

Van Sint Jan, S., 2005. Introducing anatomical and physiological accuracy in computerized anthropometry for increasing the clinical usefulness of modeling systems. Critical Reviews in Physical and Rehabilitation Medicine 17, 249-274.

Viceconti, M., Clapworthy, G. and Van Sint Jan, S., 2008. The Virtual Physiological Human - a European initiative for in silico human modelling. The Journal of Physiological Sciences 58, 441-446.

Ward, S., Eng, C., Smallwood, L. and Lieber, R., 2009. Are Current Measurements of Lower Extremity Muscle Architecture Accurate? Clinical Orthopaedics and Related Research 467, 1074-1082.

Ward, S. R., Smallwood, L. and Lieber, R. L., 2005. Scaling of Human Lower Extremity Muscle Architecture to Skeletal Dimensions. ISB XXth Congress - ASB 29th Annual Meeting. July 31 - August 5, Cleveland, Ohio.

Williams, P. E. and Goldspink, G., 1978. Changes in sarcomere length and physiological properties in immobilized muscle. Journal of anatomy 127, 459.

Winby, C. R., Lloyd, D. G. and Kirk, T. B., 2008. Evaluation of different analytical methods for subject-specific scaling of musculotendon parameters. Journal of Biomechanics 41, 1682-1688. 
Zajac, F. E., 1989. Muscle and tendon: properties, models, scaling, and application to biomechanics and motor control. Critical Reviews in Biomedical Engineering 17, 359411.

Figure 1

A) Representation of the Hill-type muscle model used in this investigation, including a contractile element (CE) connected to an elastic element in series (SE) and one in parallel (PE). B) Generic curves defining the dimensionless material properties of the tendon (left side, dashed line identifying $\tilde{\mathrm{l}}^{\mathrm{t}}=1.033$ for which $\widetilde{\mathrm{F}}^{\mathrm{m}}=1$ ) and muscle (right side, solid line: active force, dashed line: passive force, dash-dot line: total muscle force). An equilibrated isometric contraction of a muscle with fibers aligned with the tendon (zero pennation angle) is also represented (red dashed line and arrows pointing to the correspondent normalized tendon and fiber length).

Figure 2

A) Flow chart representing the main steps of the proposed algorithm for estimating musculotendon parameters. B) Flow chart representing the operations performed for assessing the results obtained from the optimization procedure. In both diagrams, musculotendon parameters produced as output by the blocks are colored as the model they are related to (blue: reference model, red: target model, green: optimized model).

Figure 3

Subject specific model generated from the LHDL dataset (downloadable at https://www.physiomespace.com) using three dimensional muscle volumes to identify muscle 
paths. The NMSBuilder model (left) and correspondent OpenSim model (right) are presented from (A) a frontal and (B) a lateral view. For ease of visualization, only selected muscles are shown in the NMSBuilder model (red: adductor magnus, green: rectus femoris, violet: tensor fasciae latae, orange: psoas major, blue: iliacus, yellow: gluteus maximus). The path of gluteus maximus was adjusted manually in the OpenSim model to correct for muscle volume flattening due to the specimen lying supine.

Figure 4

Convergence profiles of optimal fiber length, tendon slack length and root mean squared error (calculated on ten fixed points per degree of freedom) for each muscle. Percentages of variation of parameters' values estimated using $n_{d o f}$ and $n_{d o f}-1$ evaluation points per degree of freedom are represented.

Figure 5

Root mean squared errors (RMSE) (first row) and mean $( \pm \mathrm{SD})$ mapping errors (expressed as a percentage of the reference model's normalized fiber lengths) (second row) calculated on ten fixed points per degree of freedom $\left(\mathrm{n}_{\mathrm{dof}}=10\right)$ spanned by the considered muscle for Example 1 (first column) and Example 2 (second column).

Figure 6

First row: optimal fiber lengths estimated for the subject specific model compared to the measurements (mean \pm 2 SD) of Ward et al. (2009) and the values implemented in the model of Arnold et al. (2010). The only muscle outside the range of variability measured by Ward et al. (2009) (gracilis) is identified with an asterisk (see legend). Second row: tendon slack lengths calculated for the subject specific model compared to the values used by Arnold et al. (2010) and to the range of tendon lengths measured in the LHDL specimen (represented as error bars). Please note that rectus femoris tendon measurement included patellar tendon, 
tendon fraction was null in the musculotendon path of adductor brevis, gluteus medius and iliacus and no measurements were taken in the right side of the specimen for gemelli, gluteus minimus and quadratus femoris.

\section{Table 1}

Details of the presented examples of muscle parameters optimization in terms of reference model, target model, considered range of motion, number of muscles included in the model and used dimensionless muscle model. Additionally, a proportionality index to evaluate the non-uniformity of reference and target model is reported, calculated as described in Winby et al. (2008). For example 2, only pelvis, femur and tibia are used to calculate the proportionality index.

\begin{tabular}{|c|c|c|c|c|c|c|c|}
\hline Example & $\begin{array}{l}\text { Reference } \\
\text { model }\end{array}$ & Target model & $\begin{array}{l}\text { Considered range } \\
\text { (ROM) for each }\end{array}$ & $\begin{array}{l}\text { motion } \\
\text { oint }\end{array}$ & $\begin{array}{l}\text { Muscles } \\
\text { included } \\
\text { in model } \\
\end{array}$ & $\begin{array}{l}\text { Adopted } \\
\text { muscle } \\
\text { model }\end{array}$ & $\begin{array}{l}\text { Proportionality } \\
\text { index }\end{array}$ \\
\hline \multirow{6}{*}{1} & \multirow{6}{*}{ gait2392.osim } & \multirow{6}{*}{$\begin{array}{l}\text { Model } \\
\text { gait2392 } \\
\text { scaled as in } \\
\text { Hamner et al. } \\
(2010) \text {. } \\
\text { Torso and } \\
\text { right leg } \\
\text { were } \\
\text { removed. }\end{array}$} & $\begin{array}{l}\text { Maximum ROM calcul } \\
\text { left leg of running simı } \\
\text { (Hamner et al., 2010). }\end{array}$ & $\begin{array}{l}\text { ted from } \\
\text { ation }\end{array}$ & \multirow{6}{*}{$\begin{array}{l}46 \\
\text { muscles } \\
\text { of the left } \\
\text { leg. }\end{array}$} & \multirow{6}{*}{$\begin{array}{l}\text { Thelen } \\
(2003)\end{array}$} & \multirow{6}{*}{0.062} \\
\hline & & & Joint Angle & $\begin{array}{c}\text { ROM } \\
\text { [degrees] } \\
\text { min-max }\end{array}$ & & & \\
\hline & & & $\begin{array}{l}\text { hip flexion/extension } \\
\text { hip } \\
\text { abduction/adduction }\end{array}$ & $\begin{array}{c}-34-39 \\
-15-9.0\end{array}$ & & & \\
\hline & & & $\begin{array}{l}\text { hip external/internal } \\
\text { rotation }\end{array}$ & $-13-3.0$ & & & \\
\hline & & & $\begin{array}{l}\text { knee } \\
\text { flexion/extension }\end{array}$ & $-99-0.0$ & & & \\
\hline & & & $\begin{array}{l}\text { ankle } \\
\text { plantar/dorsiflexion }\end{array}$ & $-31-28$ & & & \\
\hline \multirow{5}{*}{2} & \multirow{5}{*}{$\begin{array}{c}\text { lower limb } \\
2010 \\
\text { (Arnold et al., } \\
2010 \text { ) }\end{array}$} & \multirow{5}{*}{$\begin{array}{l}\text { MRI based } \\
\text { model } \\
\text { produced } \\
\text { based on the } \\
\text { LHDL } \\
\text { dataset } \\
\text { (Viceconti et } \\
\text { al., 2008). } \\
\text { Only the } \\
\text { right leg was } \\
\text { considered. }\end{array}$} & $\begin{array}{l}\text { Maximum ROM comp } \\
\text { normal walking as repc } \\
\text { Kadaba et al. (1990). }\end{array}$ & $\begin{array}{l}\text { ible with } \\
\text { ted by }\end{array}$ & \multirow{5}{*}{$\begin{array}{l}19 \\
\text { muscles } \\
\text { (spanning } \\
\text { the right } \\
\text { hip joint) }\end{array}$} & \multirow{5}{*}{$\begin{array}{l}\text { Schutte } \\
\text { (1993) }\end{array}$} & \multirow{5}{*}{0.058} \\
\hline & & & Joint Angle & $\begin{array}{c}\text { ROM } \\
\text { [degrees] } \\
\text { min-max } \\
\end{array}$ & & & \\
\hline & & & $\begin{array}{l}\text { hip } \\
\text { flexion/extension }\end{array}$ & $-20-45$ & & & \\
\hline & & & & $-20-20$ & & & \\
\hline & & & $\begin{array}{l}\text { hip external/internal } \\
\text { rotation }\end{array}$ & $-20-20$ & & & \\
\hline
\end{tabular}



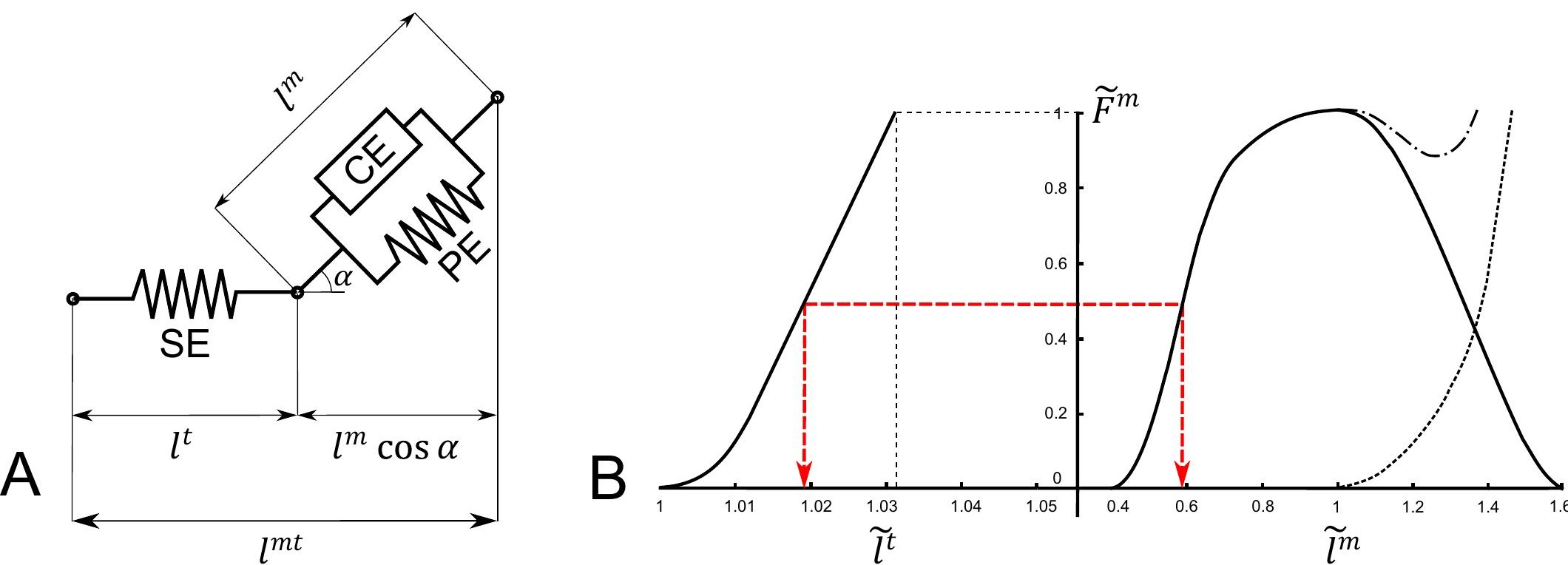


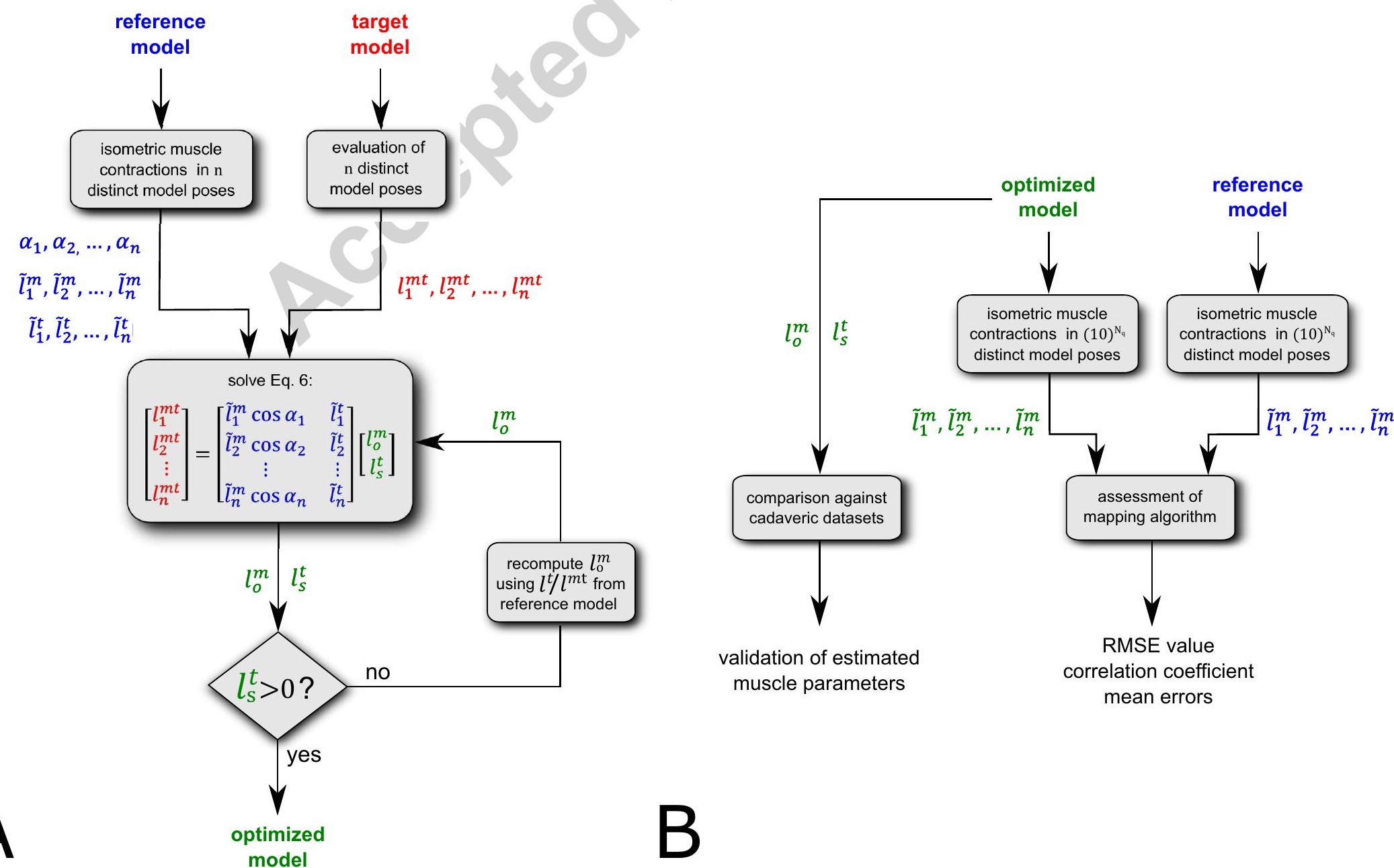



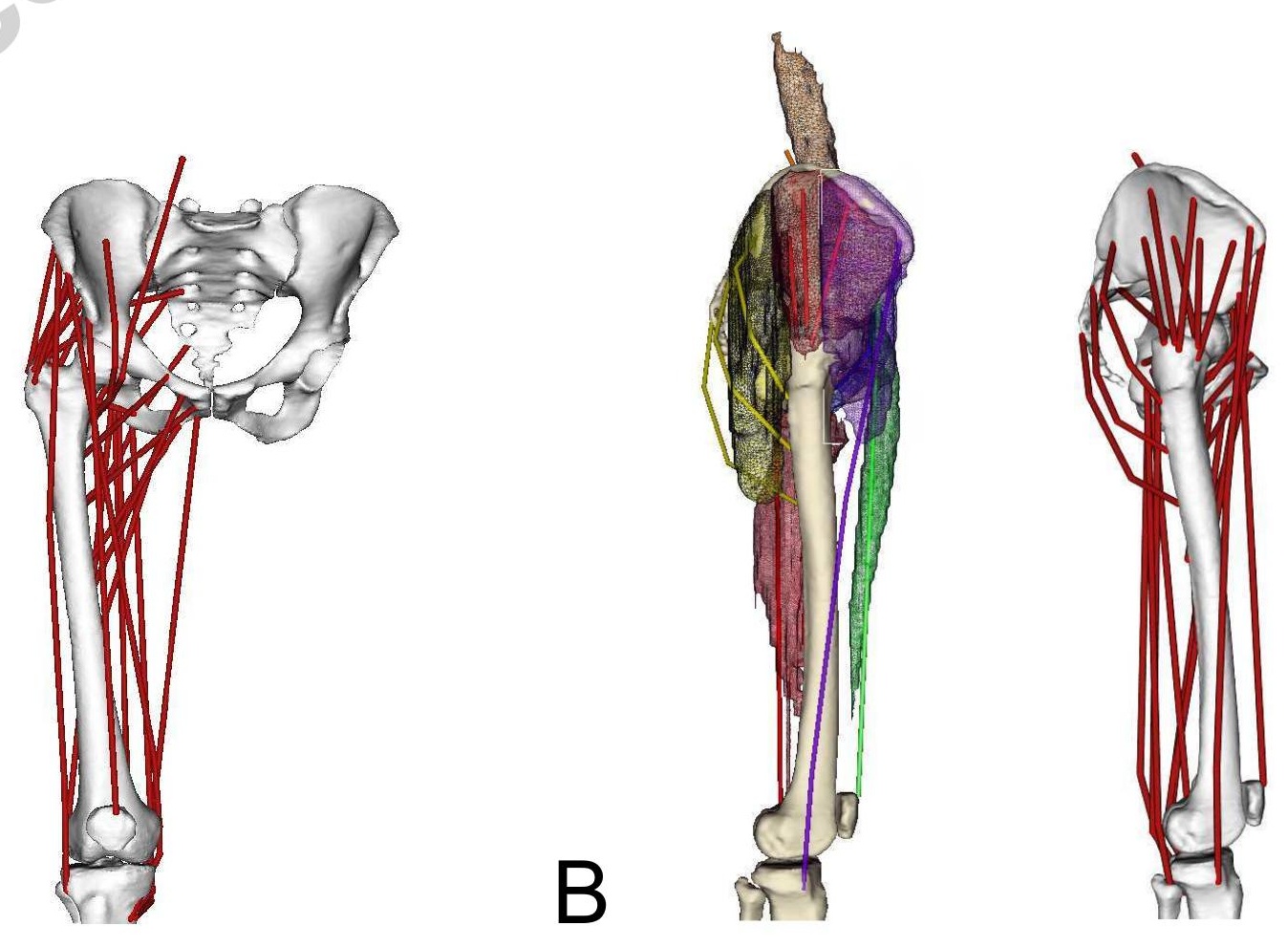

Figure 3

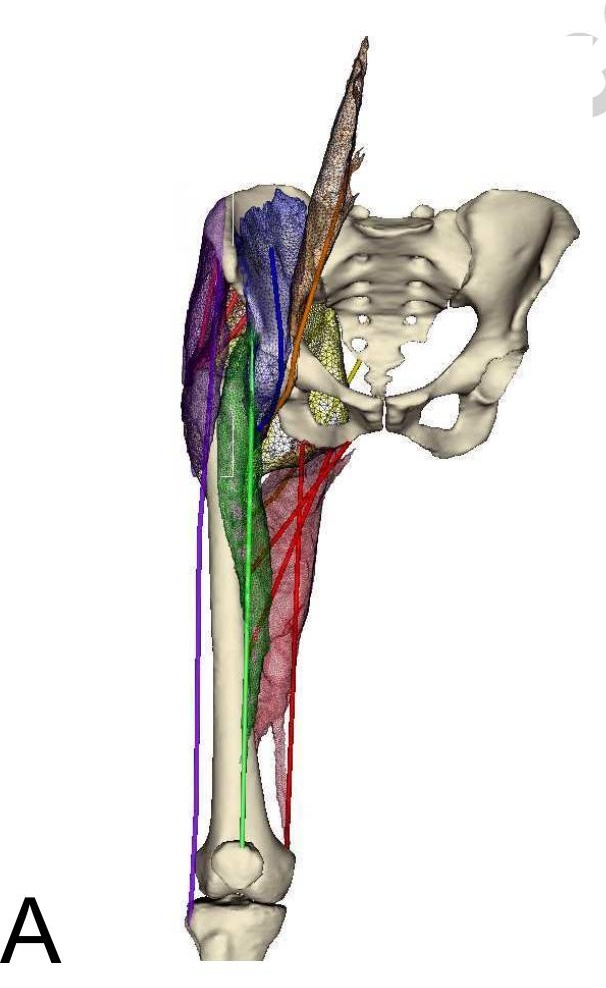

$A$ 

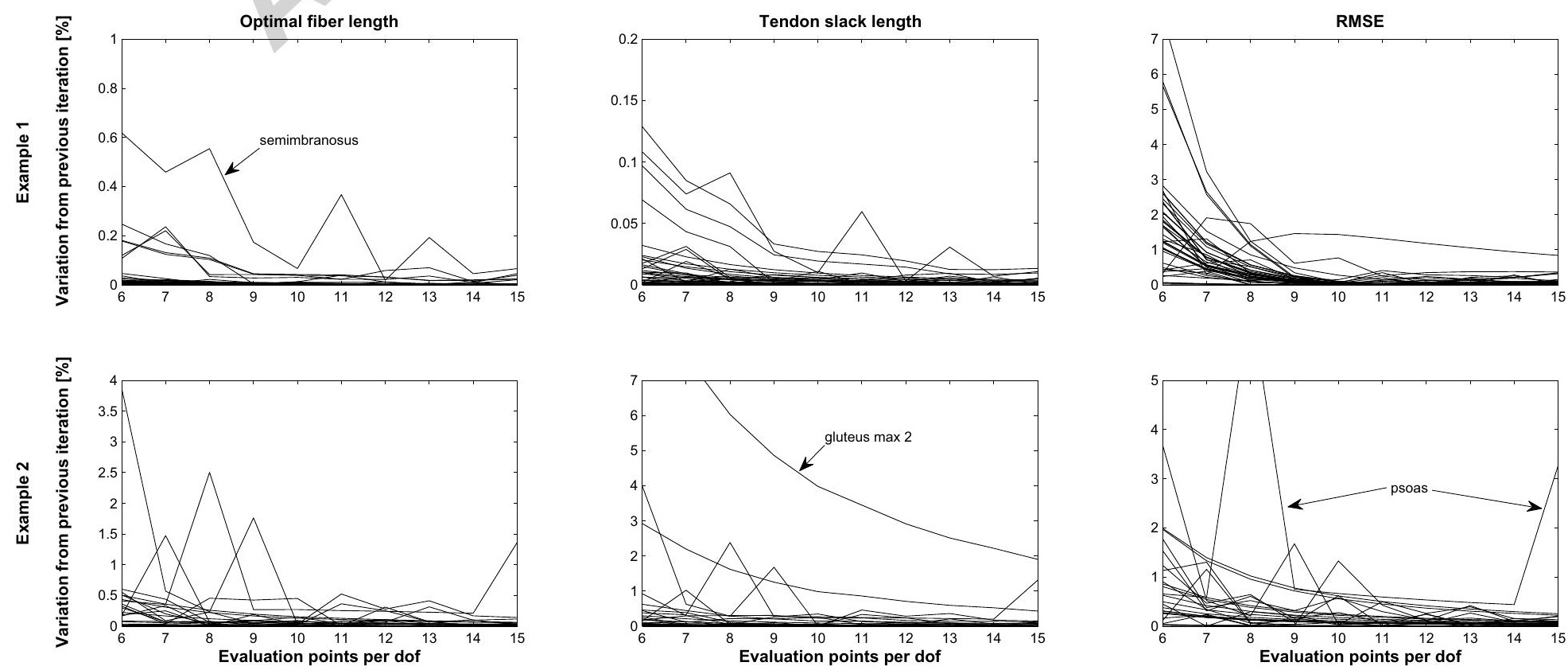


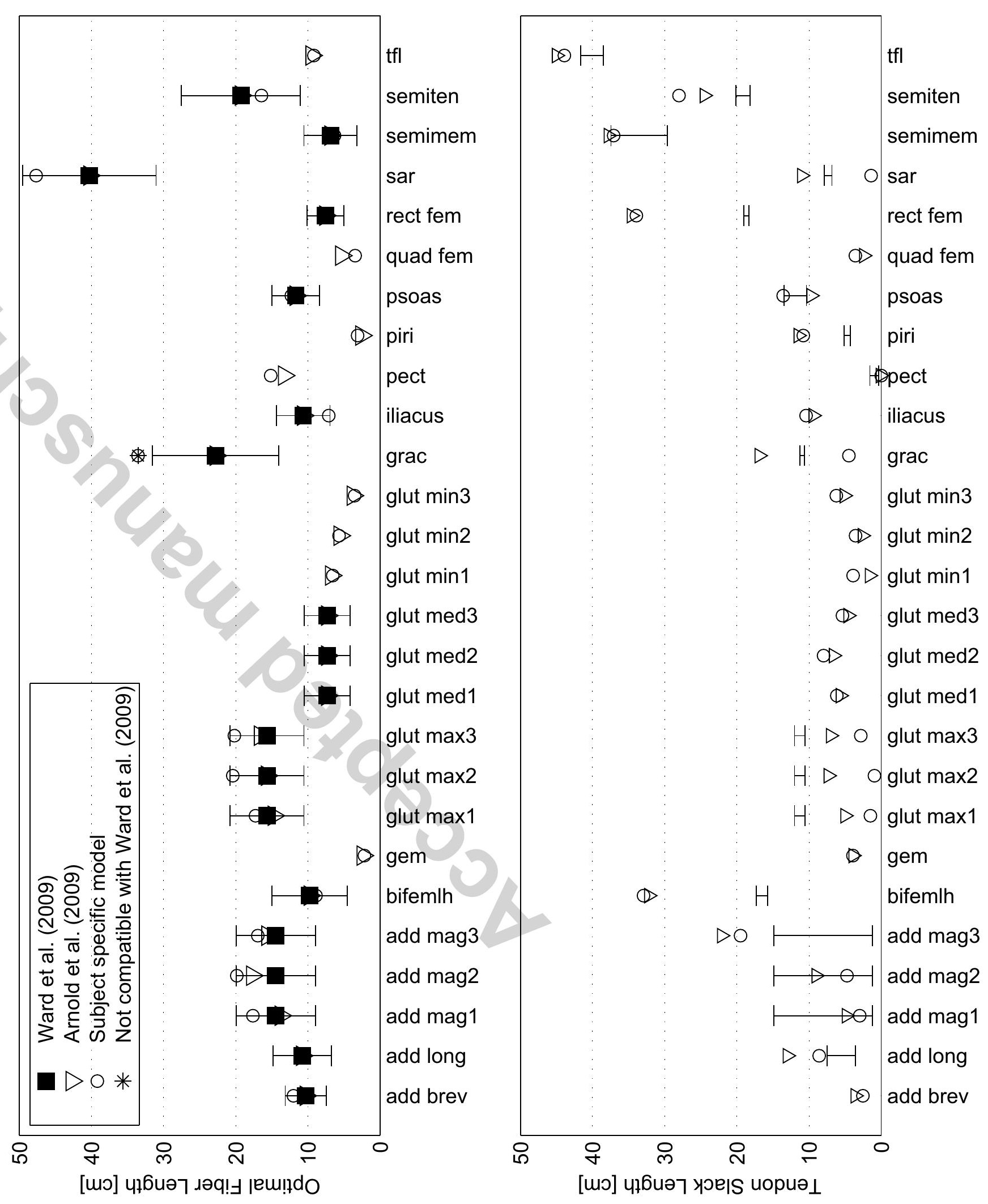




\section{Author's Accepted Manuscript}

Estimation of musculotendon parameters for scaled and subject specific musculoskeletal models using an optimization technique

Luca Modenese, Elena Ceseracciu, Monica Reggiani, David G. Lloyd

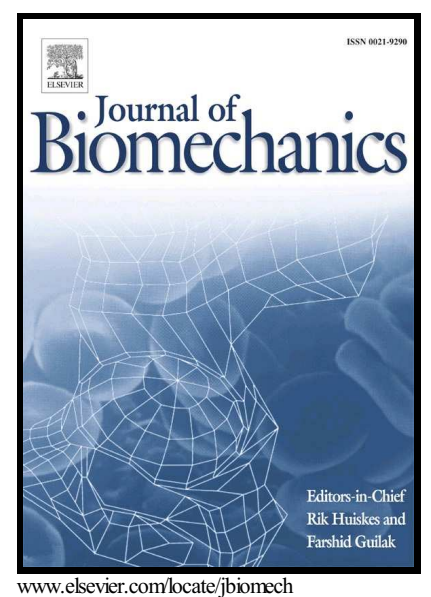

PII: $\quad$ S0021-9290(15)00636-3

DOI: $\quad$ http://dx.doi.org/10.1016/j.jbiomech.2015.11.006

Reference: BM7414

To appear in: Journal of Biomechanics

Received date: 10 June 2015

Revised date: 12 November 2015

Accepted date: 12 November 2015

Cite this article as: Luca Modenese, Elena Ceseracciu, Monica Reggiani an David G. Lloyd, Estimation of musculotendon parameters for scaled and subjec specific musculoskeletal models using an optimization technique, Journal c Biomechanics, http://dx.doi.org/10.1016/j.jbiomech.2015.11.006

This is a PDF file of an unedited manuscript that has been accepted fo publication. As a service to our customers we are providing this early version o the manuscript. The manuscript will undergo copyediting, typesetting, an review of the resulting galley proof before it is published in its final citable form Please note that during the production process errors may be discovered whic could affect the content, and all legal disclaimers that apply to the journal pertain 